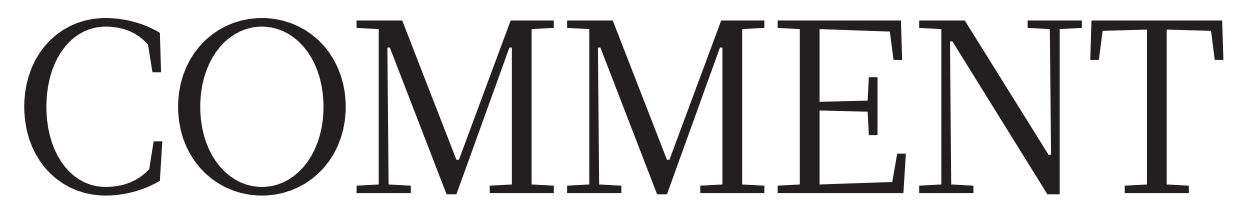

\begin{tabular}{|c|c|c|c|}
\hline $\begin{array}{l}\text { NEUROSCIENCE More big } \\
\text { thinkers weigh in on the } \\
\text { origins of creativity } \mathbf{p . 3 4}\end{array}$ & $\begin{array}{l}\text { PHYSIcS When John } \\
\text { Wheeler met Richard } \\
\text { Feynman } \mathbf{p . 4 0}\end{array}$ & $\begin{array}{l}\text { TECHNOLOGY Three views on the } \\
\text { world-changing giants of } \\
\text { Silicon Valley p.41 }\end{array}$ & $\begin{array}{l}\text { SUSTAINABILITY Work with } \\
\text { nature to withstand future } \\
\text { hurricanes p.43 }\end{array}$ \\
\hline
\end{tabular}

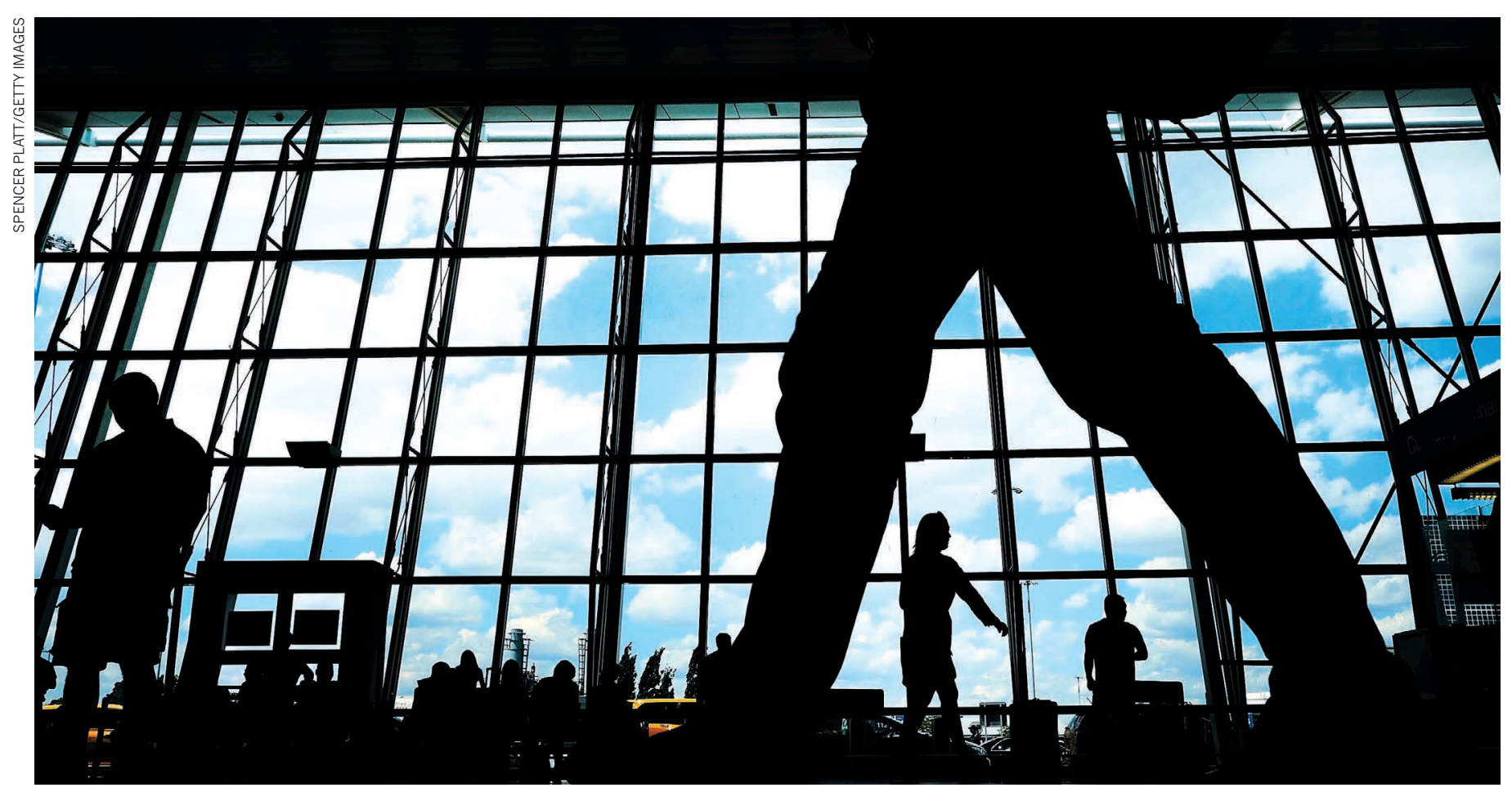

Measuring the global movements of researchers will help to assess the effects of political actions on science.

\title{
Scientists have most impact when they're free to move
}

An analysis of researchers' global mobility reveals that limiting the circulation of scholars will damage the scientific system, say Cassidy R. Sugimoto and colleagues.

$\mathrm{R}$

ecent political upheavals portend an era of increased isolationism in - science, with a chilling effect on collaboration and mobility. Last month, US President Donald Trump issued his third travel ban in a year, suspending entry of individuals from several countries into the United States, and placing restrictions on many more for visa renewals. These orders have stranded scholars abroad and prevented those who were in the country from engaging in international work. In March, UK Prime Minister Theresa May began the process of formally severing ties with the European Union. As a result, British institutions face a potential exodus of non-resident EU researchers and will have to overcome barriers to participating in and receiving funding for European collaborative projects. The list of countries engaging in these isolationist actions, and the list of actions themselves, grows longer.

To assess the impacts of such political actions, we need better ways to measure researcher mobility. Although the size and composition of the scientific workforce is fairly well established through national surveys and registries ${ }^{1}$, less is known about how often researchers move, where they go, what networks they form, and how important their movement is to the scientific impact of their work ${ }^{2}$.

We present here a new analysis based on the records of 14 million papers from nearly 16 million unique individuals who published between 2008 and 2015. In our study, some $96 \%$ of researchers had only one country of affiliation; we classed these as non-mobile. About 4\% (more than 595,000 researchers) 
$>$ were mobile - meaning that they had more than one affiliation during that period. Our analysis revealed surprising trends.

\section{CHAIN REACTION}

Over the study period, Europe and Asia saw a dramatic net loss of researchers, whereas North America saw large gains. Many commentators have anguished about 'brain drain' or 'brain gain', assuming that receiving countries get the lion's share of scientific capital at the expense of the nations from which researchers originate. The reality is more complicated (see 'Brain circulation').

We found that the majority of scientists didn't cut ties with their country of origin but instead built a chain of affiliations that linked nations together. Many researchers returned to their home country. Brain circulation may be a more apt term for the movement of contemporary scholars ${ }^{3}$.

Different nations have different roles in the circulation of elite scholars (for which our proxy is highly cited researchers). But wherever they are, wherever they stop off and wherever they come from, mobile scholars have about $40 \%$ higher citation rates, on average, than non-mobile ones (see Supplementary Information; Table S7). Closing borders takes these elite scholars out of circulation.

\section{ORIGIN STORIES}

Our study looks at the country stated in a researcher's affiliation when they published their first paper, and uses this as their country of scientific origin (this should not be confused with where they were born). We then track whether they moved or gained new affiliations in other countries over the eight years of this study.

The conventional idea of mobility focuses on migrant researchers - those who begin publishing in one country and then move to another, at some point discontinuing their attachment to the previous country. This group made up less than onethird of the mobile researchers in our study (27.3\%, or 162,519 researchers). By far the largest proportion were those we call travellers: scholars who retain a footing in their country (or countries) of scientific origin throughout their career, while gathering up more international affiliations to add to their name (72.7\%, or 433,375 researchers). Nearly half of the travellers are 'non-directional': they have more than one affiliation in their first year of publication, and they retain all these affiliations in each publication year.

Circulation networks that map the number and flow of researchers reveal the importance of the United States, United Kingdom, France, Canada and Germany as prominent nodes in the global scientific network (see Supplementary Figure S2). Isolation

of these countries would have dramatic consequences. Although the United Kingdom is not particularly central to researcher migration in the European Union, it serves a crucial function in providing a bridge for European scientists to other areas of the world (see Supplementary Figure S2). Isolationist policies in the United Kingdom could deconstruct this network, redirecting scholars through other countries.

More patterns emerge if we look only at migrants and directional travellers who moved between and within continents, who first published in 2008, and who published at least eight

papers during the study period. Admittedly, our short time window means that

\section{"Mobility is growing, complicated and increasingly threatened."} we focus on junior scholars. But it avoids conflating them with senior scholars whose movement and networking are likely to differ.

This group comprised 12,046 researchers. Europe provides the largest share (35\%) followed by about one-quarter each from Asia and North America (see 'Making tracks'). The relationship between these latter continents is strong: the majority of mobile scholars associated with an Asian address in their first publication in 2008 have a North American address by 2015, and more than one-third of mobile North American scientists end up in Asia. Both of these trends can probably be explained by the same underlying phenomenon - the influx of Asian students into the United States (some of whom publish before their arrival, and others of whom do so afterwards), and their subsequent moves back to Asia.

We see a $22 \%$ net loss of researchers from Europe, a $20 \%$ loss from Asia, and a nearly $50 \%$ gain for North America (see Supplementary Table S4). European scholars make up the largest population of mobile scholars in nearly every country, with the exception of Asian countries, where most scholars are drawn from North America (see 'Scientist shuffle').

It is also of interest to know the degree to which certain countries are responsible for the production and cultivation of highimpact scholars. We assess this by looking at citation scores for mobile researchers before and after their moves (see 'Trip adviser').

Countries in North America and Northern Europe act as strong producers: they put into circulation scholars who are wellcited before they move. The same countries are also strong cultivators, identifying talent early and providing fertile ground for scholars to achieve high impact once they arrive. Asian regions are strong recruiters, gaining affiliations with established scholars who were well-cited before their arrival.
Oceania is a notable incubator, affiliated with scholars who realize high potential once they move on.

Some migratory routes tend to be associated with highly cited scholars. Those from North America with the highest impact tend to land in Northern and Western Europe and, to a lesser extent, in Southeastern Asia. Mobile, high-impact Northern Europeans are recruited to Southern Europe; mobile, high-impact Western Europeans are recruited to Oceania and Eastern Asia.

Mobile scholars from Oceania produce particularly high-impact work when they arrive in North America and Southern Europe. Central and Western Asian scholars (including those from countries implicated in the US immigration ban) realized their highest citation rates for work done in North America and Europe. Barring scholars from these countries is likely to displace high-impact ones to other nations.

Regardless of region, mobility pays in terms of citations. Across all regions, mobile scholars are more highly cited than their non-mobile counterparts. The advantage varies by region. Mobile North Americans see only a $10.8 \%$ boost in citations over their non-mobile colleagues. For Eastern European scholars, the gulf is $172.8 \%$.

\section{MOBILITY MEASURES}

Of course, our method has limitations. We cannot tell if our 'country of academic origin' represents country of birth, of academic training or of academic employment - it reveals solely where an individual began publishing. If researchers from some countries tend not to publish first in a journal indexed in the Web of Science, then our measures would underestimate mobility from these places. Those we have counted as 'non-mobile' might really be 'pre-mobile' - meaning that they have not yet moved. Furthermore, examining less than a decade of papers, focusing on journal articles and adding restrictions for number of publications may privilege certain disciplines in the analysis. And our study does not look at mobility rates within each country: for large nations such as the United States, these might be significant and interesting.

Internationally comparable mobility indicators for the scientific workforce are particularly necessary in an era in which mobility is growing, complicated and increasingly threatened. We need indicators that provide more nuanced and dynamic assessments of the exchange of human capital and the effect of this exchange on the knowledge economy, particularly given that the 'travellers' in our analysis account for the majority of mobile scholars. This study provides a start.

It might be argued that collaboration can continue, even when mobility is restricted. 


\section{BRAIN CIRCULATION}

Countries with the strongest science systems train, keep and attract many outstanding researchers. Nations that have less-established systems realize their greatest impact by recruiting established scholars or nurturing those who go on to greatness elsewhere. Policies that reduce mobility are therefore detrimental to all.

MAKING TRACKS Intercontinental flows of scholars whose first paper was published in 2008 and who had at least 8 publications between then and 2015 .

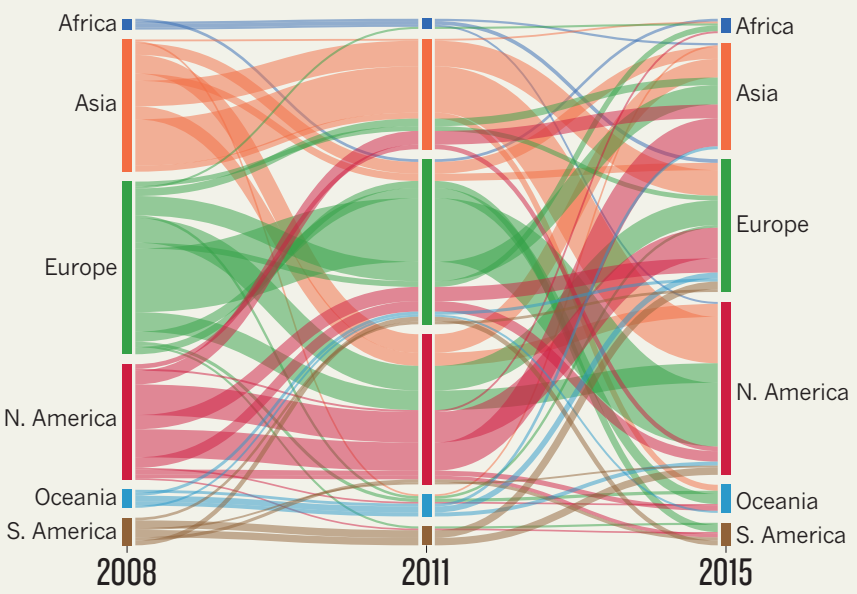

SCIENTIST SHUFFLE The proportion of mobile researchers who ended up in various regions in 2015

Region of origin in 2008

Africa Asia Europe North America Oceania South America

W Researchers returning to their original region

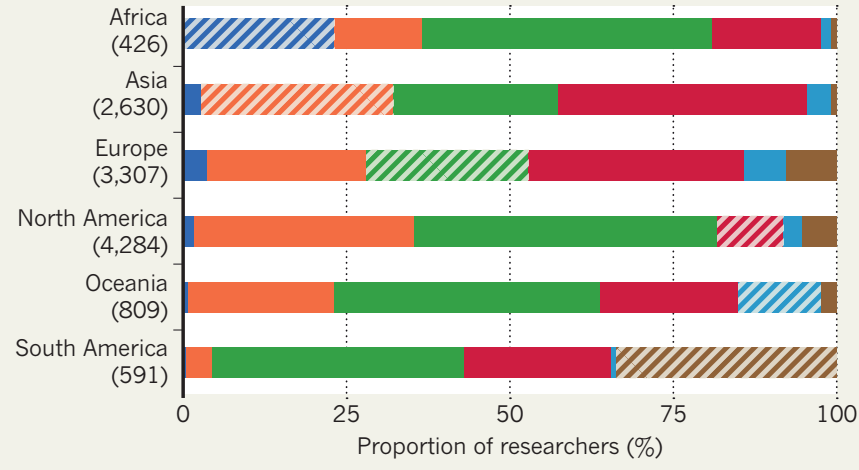

TRIP ADVISER Scientists who move country are, on average, much more highly cited than those who don't - but where they rack up citations depends on where they come from, where they stop off and where they go.
- Citations accrued here by outgoing researchers before they left. High-scoring regions are producers. - Citations accrued by incoming researchers before they arrived here. High-scoring regions are recruiters. - Citations accrued by outgoing researchers after they left here. High-scoring regions are incubators. Citations accrued here by incoming researchers once they arrived. High-scoring regions are cultivators. - Citations for scholars publishing solely in this region.

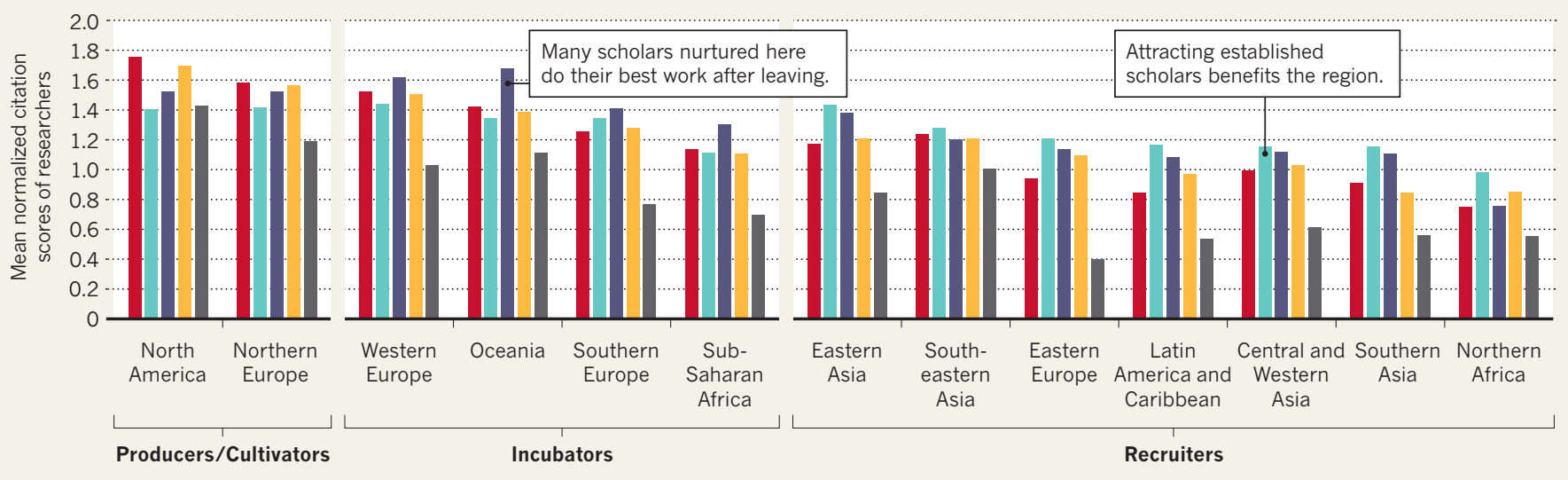

However, despite advances in computing, collaboration tends to be initiated and sustained through interactions that happen in person ${ }^{4}$. Limiting mobility is likely to have adverse effects on the scientific system, which is increasingly dependent on international collaboration ${ }^{5}$.

Disruption of the existing network would have serious effects on many nations, including large science producers and cultivators such as the United States. The country benefits significantly both from its centrality in the global knowledge network, and from the educational investments of other countries. Its exceptional contributions are disproportionately made by researchers who are both foreign-born and foreign-educated ${ }^{6}$. Disconnection would also seriously affect those nations that benefit from the United States' role as a cultivator - it invests in researchers who come from Asia and elsewhere and later return home.
Some countries may see benefits, however: isolationism among central countries can lead to advantages for other competitive nations. For example, the number of graduate-student applicants to Canada has risen since the change in the US administration (see go.nature.com/2fjc4i). However, if adequate scientific capacity does not exist in these other countries, global talent will not be cultivated to the extent it is today. With good bibliometric standards to measure the impact of mobility, we can test the effects of these political changes in the decades to come.

One thing is already clear. Internationally mobile scholars are in the minority, yet show the highest impact across the globe. Limiting the circulation of scholars will damage the entire scientific system. a SEE EDITORIAL P.7 \& COMMENT P.32

Cassidy R. Sugimoto is associate professor of informatics at Indiana University
Bloomington, USA, and a visiting professor at the Centre for Science and Technology Studies, Leiden University, Leiden, the Netherlands. Nicolas Robinson-Garcia, Dakota S. Murray, Alfredo YegrosYegros, Rodrigo Costas, Vincent Larivière.

e-mail:sugimoto@indiana.edu

1. Organisation for Economic Co-operation and Development. The Global Competition for Talent: Mobility of the Highly Skilled (OECD, 2008); available at go.nature.com/2wpaxf

2. Moed, H. F., Aisati, M. \& Plume, A. Scientometrics 94, 929-942 (2013).

3. Gaillard, J. \& Gaillard, A. M. Sci. Technol. Soc. 2, 195-228 (1997).

4. Wagner, C. S. The New Invisible College p.69 (Brookings Institution Press, 2008).

5. Adams, J. Nature 497, 557-560 (2013).

6. Stephan, P. E. \& Levin, S. G. Popul. Res. Policy Rev. 20,59-79 (2001).

Supplementary information, full author affiliations and funding details accompany this article online; see go.nature.com/2yuyhr6. 